\title{
Uso clínico de produtos do Cymbopogon citratus stapf em patologias bucais: revisão integrativa
}

\author{
Clinical use of Cymbopogon citratus stapf products in bucal pathologies: integration review \\ Uso clínico de los productos Cymbopogon citratus stapf en patologías orales: una revisión \\ integradora
}

Recebido: 10/02/2021 | Revisado: 14/02/2021 | Aceito: 02/03/2021 | Publicado: 08/03/2021

Maria Augusta Correa Barroso Magno Viana
ORCID: https://orcid.org/0000-0002-1122-1197
Universidade Federal da Paraíba, Brasil
E-mail: m.augustamagno@ hotmail.com
Caio César Ferreira Alverga
ORCID: https://orcid.org/0000-0003-1182-7364
Universidade Federal da Paraíba, Brasil
E-mail: caioalverga@ outlook.com
Gregório Márcio de Figueiredo Rodrigues
ORCID: https://orcid.org/0000-0002-9881-025X
Universidade Federal da Paraíba, Brasil
E-mail: gregorio_marcio1 @ yahoo.com.br
Laísa Vilar Cordeiro
ORCID: https://orcid.org/0000-0002-8884-7331
Universidade Federal da Paraíba, Brasil
E-mail: laisavilar@gmail.com
Edeltrudes Oliveira Lima
ORCID: https://orcid.org/0000-0002-9547-0886
Universidade Federal da Paraíba, Brasil
E-mail: edelolima@yahoo.com.br

\section{Resumo}

O presente artigo tem como objetivo apresentar uma revisão integrativa que aborde a contribuição do Cymbopogon citratus (DC.) Stapf no tratamento de Candida nas doenças da cavidade bucal. Foram selecionados artigos publicados nas bases de dados LILACS, MEDLINE/PUBMED, utilizando os descritores: Cymbopogon, Candida e candidíase bucal. Adotando como critérios de inclusão estudos que apresentaram relação com o objetivo da revisão e presença dos descritores supracitados no título, resumo e corpo do texto em português e ou inglês. A seleção dos artigos resultou em 67 estudos, os quais, 16 foram selecionados para compor a amostra, que demonstrou as formas de utilização do $C$. citratus e sua ação no tratamento da candidíase bucal. Os estudos mostram a eficácia da utilização do C. citratus no tratamento de infecções gênero Candida que acometem a cavidade bucal, principalmente como forma de complementação do tratamento. Entretanto é necessário cautela para a prescrição do uso, visto que a maioria dos estudos foram realizados em laboratórios, não sendo testados in vivo. Conclui-se que o $C$. citratus apresenta ação antifúngica contra o gênero Candida tanto na forma de óleo como de extrato, embora alguns estudos evidenciem citotoxicidade e mutagenicidade, que devem ser considerados para a segurança de sua utilização.

Palavras-chave: Candida; Candidiase bucal; Cymbopogon citratus.

\begin{abstract}
This work aims to present an integrative review on the potential of Cymbopogon citratus (DC.) Stapf on candidiasis in the oral cavity. Articles published in the LILACS, MEDLINE/PUBMED databases were selected, using the descriptors: Cymbopogon, Candida and oral candidiasis. Studies that were related to the purpose of the review and the presence of the aforementioned descriptors in the title, abstract and body of the text were included in Portuguese and or English. The selection of articles resulted in 67 studies, of which 16 were selected to compose the manuscript, which demonstrated the ways of using $C$. citratus and its action in the treatment of oral candidiasis. It was possible to demonstrate the effectiveness of using $C$. citratus to treat infections of the genus Candida that affect the oral cavity, mainly as a complement to conventional treatment. However, caution is required when prescribing use, since most studies were carried out in laboratories and have not been tested in vivo. It is concluded that both the essential oil and the extract of $C$. citratus have antifungal action against the genus Candida, although some studies show cytotoxicity and mutagenicity, which must be considered for the safety of its use.
\end{abstract}


Keywords: Candida; Buccal candidiasis; Cymbopogon citratus Stapf.

\section{Resumen}

Este artículo tiene como objetivo presentar una revisión integradora que aborde la contribución de Cymbopogon citratus (DC.) Stapf en el tratamiento de Candida en enfermedades de la cavidad oral. Se seleccionaron los artículos publicados en las bases de datos LILACS, MEDLINE / PUBMED, utilizando los descriptores: Cymbopogon, Candida y candidiasis oral. Adoptando como criterios de inclusión estudios que tuvieran relación con el propósito de la revisión y la presencia de los descriptores antes mencionados en el título, resumen y cuerpo del texto en portugués y o en inglés. La selección de artículos resultó en 67 estudios, de los cuales 16 fueron elegidos para componer la muestra, que demostraron las formas de uso de C. citratus y su acción en el tratamiento de la candidiasis oral. Los estudios demuestran la efectividad del uso de C. citratus en el tratamiento de infecciones del género Candida que afectan la cavidad bucal, principalmente como forma de complementar el tratamiento. Sin embargo, se requiere precaución al prescribir el uso, ya que la mayoría de los estudios se llevaron a cabo en laboratorios y no se han probado in vivo. Se concluye que $\mathrm{C}$. citratus tiene acción antifúngica contra el género Candida tanto en forma de aceite como de extracto, aunque algunos estudios muestran citotoxicidad y mutagenicidad, lo que debe considerarse para la seguridad de su utilización.

Palabras clave: Candida; Candidiasa oral; Cymbopogon citratus.

\section{Introdução}

O processo infeccioso denominado candidíase ou candidose é causado for fungos do gênero Candida, manifestandose de modo oportunista no hospedeiro em três grupos de acordo com o local de envolvimento: mucocutânea (atingindo mucosa oral ou vaginal com ulcerações e placas edemaciadas), cutânea (pele) e sistêmica, onde há comprometimento de vários órgãos e/ou sistemas que nesse último pode elevar o indivíduo a óbito (Melo \& Guerra, 2014).

Fungos são micro-organismos que se organizam em comunidades estruturadas funcionais, com sistema coordenado formando biofilmes que estão associadas com patologias na cavidade bucal. Espécies do gênero Candida estão presentes em $20 \%$ a 50\% dos indivíduos dentados saudáveis e vem sendo bastante associadas a essas lesões orais, em especial Candida albicans, porém, outras espécies como C. tropicalis, C. glabrata e C. krusei, também são identificadas com frequência (Melo \& Guerra, 2014).

A candidíase bucal é a infecção fúngica oportunista mais comum em indivíduos infectados com o vírus da imunodeficiência humana (HIV), sendo objeto de grande estudo os constituintes sintéticos ou biológicos para controle do biofilme formado por este patógeno. Esse tipo de infecção geralmente é detectado por sinais clínicos, necessitando de testes mais específicos por culturas de sangue, embora ainda seja um desafio para os médicos e microbiologistas (Melo \& Guerra, 2014; Spalanzani, et al., 2018).

Pacientes com candidíase bucal são frequentemente tratados com nistatina ou miconazol, antifúngicos utilizados de modo tópico para tratamento de infecções superficiais, ao passo que outros agentes como fluconazol, itraconazol ou voriconazol são indicados para o tratamento de infecções profundas, como nos casos de candidíase bucal recalcitrante quando o tratamento tópico falha. No entanto, a susceptibilidade reduzida de C. glabrata aos azoles pode ser um problema para o tratamento de infecções causadas por esta espécie, portanto é necessário estudo constante de novas substâncias para combater a resistência desses fungos (Sharifzadeh, et al, 2013; Swetha, et al., 2016; Miranda-Cadena, et al., 2018).

Uma opção para o tratamento da candidíase bucal é o uso de fitoterápicos. Esses são medicamentos compostos por várias substâncias encontradas nas "espécies vegetativas" preparadas segundo a legislação vigente, tendo como apresentação farmacêutica as cápsulas, géis, infusões, soluções aquosas e hidroalcoólicas, comprimidos e pomadas. Essas substâncias são constantemente estudas para a elaboração de fármacos antimicrobianos, porém a maioria desses tem em suas bases moléculas oriundas de extratos de plantas, fortalecendo a necessidade do estudo de fitoterápicos para a descoberta do princípio ativo e sua ação contra microorganismos (Machado \& Oliveira, 2014). 
Estudos evidenciam o efeito do tratamento contra candidíase bucal ao utilizar produtos fitoterápicos tendo como base a espécie Cymbopogon citratus (DC.) Stapf, conhecida popularmente como erva-cidreira, capim-limão e capim-cidreira (Silva et al, 2017; Prakash, et al., 2016). C. citratus é uma planta perene encontrada em várias localidades do mundo, principalmente em regiões tropicais e subtropicais. Sua utilização na forma de extrato e óleo essencial tem sido feita há décadas devido a sua ampla atividade farmacológica e terapêutica (Ekpenyonga \& Akpan, 2015).

Do ponto de vista etnofarmacológico, Cymbopogon citratus (DC.) Stapf tem sido utilizado na medicina popular como carminativo, sedativo, sudorífero, febrífugo, diurético, antipirético e antireumático. Além disso, pesquisas constataram que essa espécie também pode ser usada como: calmante e espasmolítico leve, analgésico, diurético, anticarcinogênica, antibacteriana, antihelmítica, antifúngica, inseticida e larvicida, que são atribuídas aos compostos a-citral, â-citral e mirceno do óleo essencial (Lemos, et al., 2013; Pokpong, et al., 2014; Boukhatem, et al., 2014).

Diante do exposto, procura-se oferecer o entendimento da utilização de produtos do Cymbopogon citratus (DC.) Stapf contra leveduras do gênero Candida e consequentemente demonstrar a efetividade no âmbito do tratamento para a candidíase oral, sendo este uma possível forma de tratamento substitutivo aos medicamentos sintéticos, ou uma forma complementar, auxiliando a enfrentar dificuldades do tratamento com medicamentos sintéticos utilizados há um prolongado tempo, como o aumento à tolerância - e diminuição do efeito, e ou efeitos colaterais assíduos provenientes dos medicamentos sintéticos, sendo possível assim, uma diminuição da quantidade prescrita e um correlata mitigação ao indivíduo. Portanto, o presente artigo tem como objetivo apresentar uma revisão integrativa que aborde a contribuição do C. citratus no tratamento de candidíases na cavidade bucal.

\section{Metodologia}

Trata-se de uma revisão integrativa da literatura, de natureza qualitativa, sobre diferentes abordagens referentes quanto ao uso de Cymbopogon citratus (DC.) Stapf no tratamento de candidíase bucal. Segundo Mendes et al. (2008), a revisão integrada é um recurso da prática baseada em evidências (PBE) que permite a síntese e análise de estudos produzidos referente à temática investigada. Consiste em uma técnica de pesquisa que apresenta rigor metodológico, ampliando a confiabilidade e a profundidade das conclusões da revisão (Pereira et al., 2018).

A questão norteadora admitida para esta pesquisa foi: quão eficaz é o tratamento da candidíase oral ao utilizar produtos derivados de C. citratus.

A coleta dos dados foi realizada no período de julho/2018 a julho/2019, através de buscas nas bases de dados eletrônicas da LILACS (Literatura Latino-Americana em Ciências da Saúde), e MEDLINE/PUBMED (US National Library of Medicine's - NLM), com a utilização da combinação dos Descritores em Ciências da Saúde (DeCS) em português e em inglês: Candidal Candida, Candidiase bucal/ Candidiasis oral, Cymbopogon/ Cymbopogon.

A busca dos artigos foi realizada através das combinações dos DeCS utilizando os conectivos booleanos para fazer as associações. As combinações utilizadas foram:

1. Candida AND Cymbopogon;

2. Candidiase bucal AND Cymbopogon;

3. Candida AND “Cymbopogon”;

4. Oral candidiases AND Cymbopogon. 
A inclusão dos artigos foi definida em três etapas: i) triagem por meio da leitura do título, ii) leitura do resumo, iii) leitura na íntegra. Foram inclusos estudos publicados nos últimos 11 anos considerando tanto estudos in vitro como in vivo. Os critérios de inclusão estabelecidos foram: estudos que apresentaram relação com o objetivo da revisão e presença dos descritores supracitados no título, resumo, corpo do texto. Como critério de exclusão foram considerados estudos repetidos nas bases de dados utilizadas e pesquisas de revisão da literatura.

A princípio foram selecionadas 67 publicações, sendo excluídas aquelas que não atendiam aos critérios de inclusão. Após aplicação dos critérios de inclusão e leitura prévia foram selecionadas 16 publicações, consideradas relevantes para o objeto de estudo e discussão acerca da problemática em pauta. Das publicações selecionadas 4 foram da base de dados LILACS e 12 da MEDLINE/PUBMED. A estratégia de busca foi realizada conforme sistematizada na Figura 1.

Ressalta-se que todas as informações obtidas estão respaldadas pelo registro das referências dos autores ao final do trabalho, respeitando, assim, os aspectos éticos.

Figura 1 - Fluxograma da estratégia de busca para a seleção dos estudos. João Pessoa, Paraíba, Brasil, 2021.

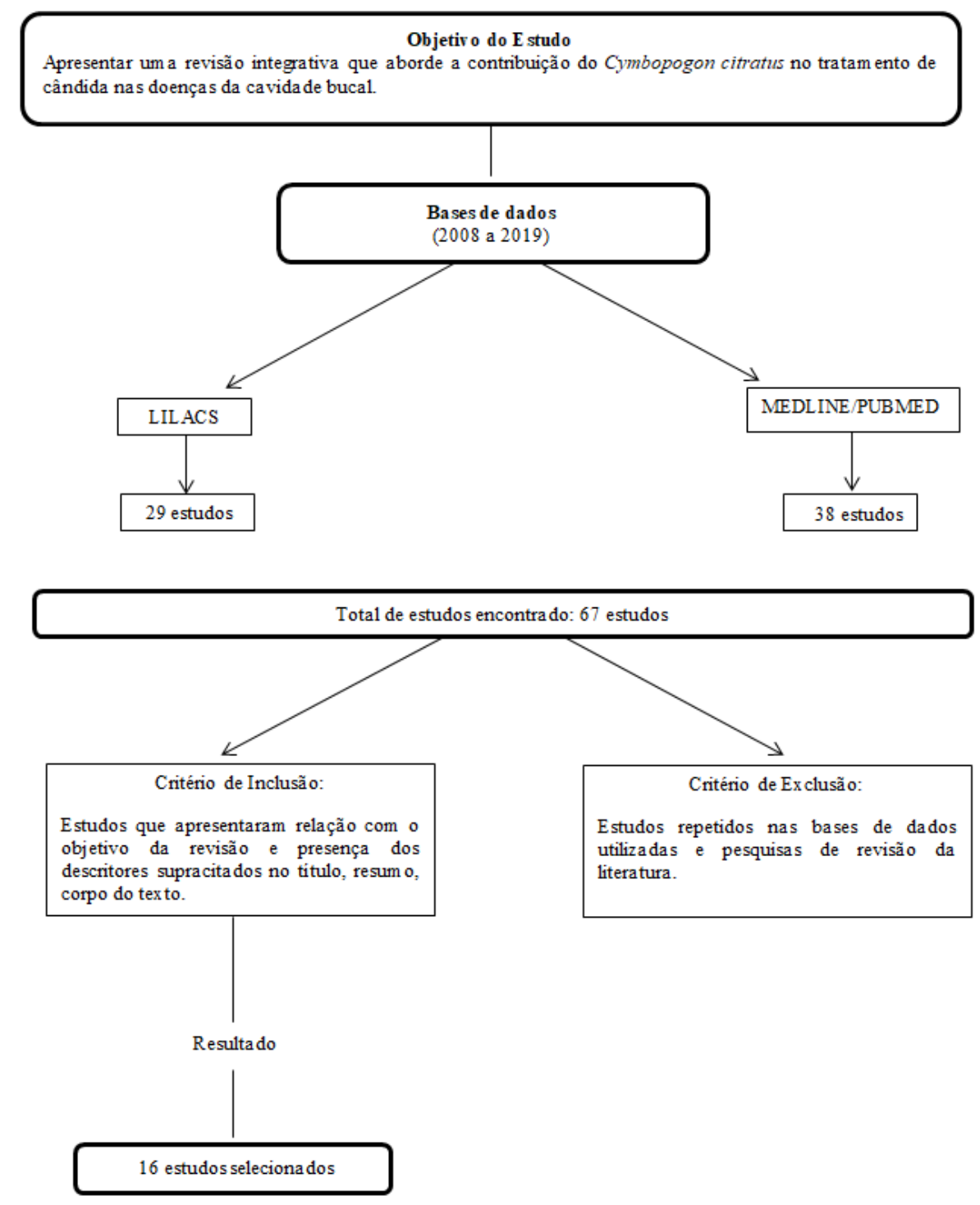

Fonte: Autores. 
Podemos observar no fluxograma acima como foi à organização da busca dos dados, sendo consideradas as publicações entre os anos de 2008 a 2019, e assim, respeitando a temática e as combinações dos descritores utilizadas. De início foram selecionados 29 estudos na base de dados LILACS e 38 estudos na base de dados MEDLINE/PUBMED e após passar pelos critérios de inclusão e exclusão permaneceram 16 estudos.

\section{Resultados e Discussão}

Os dados essenciais dos estudos selecionados foram sintetizados e dispostos no Quadro 1 contendo: referências, as principais abordagens dos estudos e seus resultados. As análises dos dados foram realizadas de forma descritiva, caracterizadas conforme a extração dos dados selecionados a partir da identificação das variáveis de interesse referente à temática: Cymbopogon citratus (DC.) Stapf no tratamento de candidíase bucal.

Quadro 1 - Estudos que utilizaram o Cymbopogon citratus no tratamento de cândida nas doenças da cavidade bucal.

\begin{tabular}{|c|c|c|c|}
\hline Referência & $\begin{array}{c}\text { Material/ parte da } \\
\text { planta }\end{array}$ & CEPAS & Principais resultados \\
\hline $\begin{array}{c}\text { Koseki \& Tanaka \& } \\
\text { Murata, } 2018\end{array}$ & óleo & C. albicans ATCC 18804 & $\begin{array}{l}\text { Foi observado um CIM para o óleo } \\
\text { de capim-limão de } 0,0625 \% \text { e um } \\
\text { CFM de } 0,125 \% \text { nas cepas de } C \text {. } \\
\text { albicans ATCC } 18804 \text {. Os } \\
\text { resultados demonstram que o óleo } \\
\text { de capim-limão tem efeito } \\
\text { antifúngico contra } C \text {. albicans. }\end{array}$ \\
\hline Juniatik, et al., 2017 & óleo/ caule e folhas & C. albicans ATCC 10231 & $\begin{array}{l}\text { A nanoemulsão, ou seja, junção de } \\
\text { óleos (óleo de campim-limão com } \\
\text { óleo de limão Kaffir) tem atividade } \\
\text { antifúngica de } 83,76 \% \text { contra } C \text {. } \\
\text { albicans, sendo eficaz na inibição } \\
\text { do crescimento da } C \text {. albicans. }\end{array}$ \\
\hline Silva, et al., 2017 & óleo & $\begin{array}{l}\text { Candida albicans } \\
\text { ATCC } 90029 \\
\text { C. albicans } \\
\text { CBS } 562\end{array}$ & $\begin{array}{l}\text { O óleo essencial apresentou efeito } \\
\text { de inibição moderada conforme } \\
\text { CIM ( } 100 \text { e } 500 \mu \mathrm{g} / \mathrm{mL}), \\
\text { proporcionando efeito, fungicida. }\end{array}$ \\
\hline Madeira, et al., 2016 & extrato & C. albicans & $\begin{array}{l}\text { Os resultados demonstram que o } \\
\text { extrato do capim-limão obteve } \\
\text { CIM de } 0,625 \mathrm{mg} / \mathrm{ml} \text { e CFM de } \\
2,5 \mathrm{mg} / \mathrm{ml} \text { em cepas de } C \text {. albicans } \\
\text { em biofilmes que estavam em } \\
\text { próteses. Podendo concluir que a } \\
\text { imersão da superfície da prótese no } \\
\text { extrato do capim-limão foi eficaz } \\
\text { na redução de biofilmes de } C \text {. } \\
\text { albicans. }\end{array}$ \\
\hline Prakash, et al., 2016 & extrato/ folhas & $\begin{array}{l}\text { C. albicans } \\
\text { MTCC } 183 \\
\text { C. tropicalis } \\
\text { MTCC } 184 \\
\text { C. glabrata } \\
\text { MTCC } 3984\end{array}$ & $\begin{array}{l}\text { O extrato metanólico de } C \text {. citratus } \\
\text { mostrou atividade inibitória apenas } \\
\text { contra } C \text {. albicans }(8,2 \mathrm{~mm}) .\end{array}$ \\
\hline
\end{tabular}




\begin{tabular}{|c|c|c|c|}
\hline Pavithra, et al., 2014 & extrato & C. albicans & $\begin{array}{l}\text { O óleo de capim-limão demonstrou } \\
\text { resultado significativo para } \\
\text { diminuição da adesão da } C \text {. } \\
\text { albicans, servindo como agente } \\
\text { antiaderente em células epiteliais } \\
\text { orais em pacientes com HIV. }\end{array}$ \\
\hline $\begin{array}{l}\text { Pokpong, et al., } \\
2014\end{array}$ & óleo & C. albicans ATCC 10231 & $\begin{array}{l}\text { O óleo essencial de capim-limão } \\
\text { apresentou potente atvidade } \\
\text { antifúngica contra } C \text {. albicans } \\
\text { ATCC } 10231 \text { e seu valor de CIM } \\
\text { foi } 0,06 \%(0.625 \mu 1 / \mathrm{ml}) \text {. O óleo de } \\
\text { capim-limão apresentou uma } \\
\text { eficácia anticandidial, } \\
\text { minimizando e eliminando o } \\
\text { crescimento de fungos. }\end{array}$ \\
\hline $\begin{array}{l}\text { Boukhatem, } \\
\text { et al., } 2014\end{array}$ & óleo/ folhas & C. albicans & $\begin{array}{l}\text { Conforme resultados do ensaio de } \\
\text { difusão de vapor, ambas as } \\
\text { leveduras foram inibidas } \\
\text { completamente pelos vapores da } \\
\text { LGEO em } 60 \mathrm{ml} \text { por disco. }\end{array}$ \\
\hline $\begin{array}{l}\text { Almeida, } \\
\text { et al., } 2013\end{array}$ & óleo/ folhas & C. albicans & $\begin{array}{c}\text { Óleo essencial de } C \text {.citratus } \\
\text { apresentou atividade fungistática às } \\
\text { cepas de C. albicans. CIM igual a } \\
0,062 \% \text {. }\end{array}$ \\
\hline $\begin{array}{l}\text { Almeida, } \\
\text { et al., } 2013\end{array}$ & óleo/ folhas & C. glabrata & $\begin{array}{l}\text { Para C. glabrata } 50 \% \text { das cepas } \\
\text { apresentaram CIM de } 0,062 \% \text { de } \\
\text { óleo essencial. }\end{array}$ \\
\hline $\begin{array}{l}\text { Taweechaisup, et al., } \\
2012\end{array}$ & óleo & C. dubliniensis & $\begin{array}{l}\text { O óleo do capim-limão apresentou } \\
\text { CIM de } 0,43 \mathrm{mg} / \mathrm{ml} \text { e um CFM de } \\
0,86 \mathrm{mg} / \mathrm{ml} \mathrm{em} \text { cepas de Cândida } \\
\text { dubliniensis em biofilmes. Estes } \\
\text { resultados demonstram que o } \\
\text { capim-limão possui propriedades } \\
\text { antifúngicas e atividades } \\
\text { antibiofilme. }\end{array}$ \\
\hline Khan \& Ahmad, 2012 & óleo & $\begin{array}{l}\text { C. albicans CA01-18; } \\
\text { C. glabrata CG01,02; } \\
\text { Candida albicans } \\
\text { MTCC183; } \\
\text { Candida glabrata } \\
\text { MTCC } 3019\end{array}$ & $\begin{array}{c}\text { Os resultados demonstram } \\
\text { atividade antibiofilme promissora } \\
\text { para o capim-limão, confirmando o } \\
\text { porquê do seu uso } \\
\text { etnofarmacológico deste óleo em } \\
\text { mucocutâneas por Candida. Além } \\
\text { disso, sugere a exploração deste } \\
\text { óleo como base para novos } \\
\text { produtos na perspectiva de lidar } \\
\text { com cepas resistentes a drogas, } \\
\text { como é o caso das cepas utilizadas } \\
\text { neste estudo. }\end{array}$ \\
\hline $\begin{array}{l}\text { Taweechaisup, et al., } \\
2012\end{array}$ & óleo & C. albicans & $\begin{array}{l}\text { Foi apresentado neste estudo um } \\
\text { CIM e CFM de } 0,4 \mathrm{mg} / \mathrm{ml} \text { - } 0,8 \\
\mathrm{mg} / \mathrm{ml} \text { respectivamente. Observa- } \\
\text { se com estes resultados que o óleo } \\
\text { de capim-limão demonstra efeito } \\
\text { antibiofilme e pode modular a } \\
\text { colonização por candida. }\end{array}$ \\
\hline
\end{tabular}




\begin{tabular}{|c|c|c|c|}
\hline Tyagi, et al., 2010 & óleo & $\begin{array}{c}\text { C. albicans } \\
\text { ATCC } 10231\end{array}$ & $\begin{array}{l}\text { Uma concentração de } 288 \mathrm{mg} / \mathrm{l} \text { de } \\
\text { óleo essencial de capim-limão foi } \\
\text { suficiente para inibição } \\
\text { completa do crescimento de } C \text {. } \\
\text { albicans. } \\
\text { O óleo essencial de capim-limão } \\
\text { inibiu crescimento, como é } \\
\text { evidente pelo diâmetro de } 90 \mathrm{~mm} \\
\text { da zona de inibição, } \\
\text { proporcionando efeito, fungicida. }\end{array}$ \\
\hline Irkin, et al., 2009 & óleo & $\begin{array}{l}\text { C. albicans } \\
\text { ATCC10231 } \\
\text { C. albicans } \\
\text { C. oleophila }\end{array}$ & $\begin{array}{l}\text { Óleo essencial de capim-limão teve } \\
\text { um efeito inibitório sobre todas as } \\
\text { leveduras. Com base no resultado } \\
\text { do CIM, o óleo apresentou } \\
\text { potencial antifúngico. A maioria } \\
\text { das cepas foram inibidas com } \\
\text { valores maiores que } 0,5 \mathrm{~L} / \mathrm{mL} \text { do } \\
\text { CFM, proporcionando efeito, } \\
\text { fungicida. }\end{array}$ \\
\hline $\begin{array}{l}\text { Almeida, } \\
\text { et al., } 2008\end{array}$ & extrato/ folhas & C. albicans & $\begin{array}{l}\text { A CIM do extrato de } C \text {. citratus foi } \\
\text { de } 31,25 \mathrm{mg} . \mathrm{mL}-1 \text { para } 56,6 \% \text { das } \\
\text { cepas de } C \text {. albicans; A CFM do } \\
\text { extrato foi de } 62,5 \mathrm{mg} . \mathrm{mL}-1 \text { para } \\
96,6 \% \text { das cepas de } C \text {. albicans, } \\
\text { proporcionando efeito fungistático. }\end{array}$ \\
\hline $\begin{array}{l}\text { Almeida, } \\
\text { et al., } 2008\end{array}$ & extrato/ folhas & $\begin{array}{c}\text { C. glabrata } \\
\text { C. parapsilosis }\end{array}$ & $\begin{array}{l}\text { A CIM do extrato de } C \text {. citratus foi } \\
\text { de } 62,5 \mathrm{mg} . \mathrm{mL}-1 \text { para } 100 \% \text { das } \\
\text { cepas de } C \text {. glabrata, } C \text {. } \\
\text { parapsilosis e } C \text {. krusei; A CFM } \\
\text { do extrato foi de } 125 \mathrm{mg} . \mathrm{mL}-1 \\
\text { para } 100 \% \text { das cepas de } C . \\
\text { glabrata, } C \text {. parapsilosis, } \\
\text { proporcionando efeito fungistático. }\end{array}$ \\
\hline Silva, et al., 2008 & óleo & $\begin{array}{c}\text { C. albicans } \\
\text { ATCC 10231; } \\
\text { C. albicans } \\
\text { ATCC 18804; } \\
\text { C. albicans } \\
\text { CI-I (isolado clínico); } \\
\text { C.albicans CI-II; } \\
\text { C. glabrata } \\
\text { ATCC 2001; } \\
\text { C. parapsilosis } \\
\text { ATCC } 22019\end{array}$ & $\begin{array}{l}\text { De acordo com o resultado do } \\
\text { método de difusão de disco o óleo } \\
\text { exibiu ação contra todos os } \\
\text { Candida spp., espécies de } C \text {. } \\
\text { albicans, apresentou inibição } \\
\text { parcial quando utilizou-se um } \\
\text { volume de } 8,0 \mu \mathrm{L} \text { de cada amostra. }\end{array}$ \\
\hline
\end{tabular}

CIM: Concentração inibitória mínima; CFM: Concentração fungicida mínima. Fonte: Autores.

O quadro acima mostra o que vem sendo discutido cientificamente a respeito do uso do Cymbopogon citratus com ênfase no tratamento de cândida nas doenças da cavidade bucal. Diante desses resultados observou-se que a utilização de produtos derivados do C. citratus encontram-se na forma de óleo ou extrato, a maioria obtida por meio das folhas da planta ou adquirida por empresas. As leveduras utilizadas foram provenientes de isolados clínicos e/ou cepas padrão. Sua eficácia foi avaliada conforme os resultados da Concentração Inibitória Mínima (CIM- $\mu \mathrm{g} / \mathrm{mL})$, e pela Concentração Fungicida Mínima 
(CFM- $\mu \mathrm{g} / \mathrm{mL})$. Todos os estudos foram feitos in vitro. Sendo 31,25\% (5/16), realizados no continente Asiático, 31,25\% (5/16), no continente Africano e 6,25\% (1/16), no Europeu. No Brasil registrou 31,25\% (5/17) dos estudos relacionados com a temática da presente pesquisa.

Nos estudos encontrados, os resultados demonstraram que o extrato ou óleo advindo das folhas da C. citratus, têm efeito fungicida e ou fungistático contra as leveduras: Candida albicans, Candida glabrata, Candida oleophila, $e$ Candida parapsilosis (Silva, et al., 2017; Prakash, et al., 2016; Pokpong, et al., 2014; Boukhatem, et al., 2014; Almeida, et al., 2013; Tyagi, et al., 2010; Almeida, et al., 2008; Silva, et al., 2008).

Segundo Crocco et al. (2004) a atividade antifúngica da planta $C$. citratus deve-se ao citral, componente predominante do óleo obtido das folhas, além dele, o óleo também é constituído em boas quantidades por geraniol e $\beta$-mirceno.

No Quadro 1, há resultado demonstrando que o extrato metanólico do C. citratus teria efeito inibitório sobre a Candida albicans, porém, Bhatti et al. (2018), em um estudo mais recente, também utilizou o extrato metanólico a partir das folhas do $C$. citratus, e não houve nenhum resultado positivo para a correlação com a Candida albicans. Fornece-se então, insights para novos estudos, sobre quais as variantes de C. albicans que poderiam ter resultados significativos, e quais os métodos mais assertivos para produção do extrato.

É observado que $C$. citratus tem atividade antifúngica baseado em diversos estudos que seguem uma linha do tempo apresentando resultados significativos, sendo interessante comparar os resultados obtidos a partir desta planta, frente a medicamentos utilizados na terapia tradicional para casos de candidíase bucal. Nesse contexto, Dutta et al. (2007), constataram em seu estudo, que o óleo proveniente das folhas do C. citratus provocou o dobro de zona de inibição por microlitros $(21.17 \mathrm{~mm})$ quando correlacionado ao miconazol em $1000 \mu \mathrm{g} / \mathrm{ml}(1.44 \mathrm{~mm})$ e ao clotrimazol em $1000 \mu \mathrm{g} / \mathrm{ml}(1.39 \mathrm{~mm})$, demonstrando assim um efeito antifúngico bastante significativo contra o gênero Candida.

Corroborando com esse resultado, uma pesquisa mais recente de Khosravi et al. (2018), comparou diversos óleos advindos de plantas com potencial antifúngico, entre esses, o C. citratus, e relatou que os componentes naturais realmente têm efeito anticandidial, no entanto, seria mais seguro utilizá-los como agentes complementares à terapia tradicional, ou seja, aos medicamentos, possibilitando a diminuição da dose recomendada. Quando avaliado formas de terapias tópicas como gel, Taweechaisupapong et at. (2018), observaram, em um modelo animal, um gel formulado do óleo do C. citratus, com o gel Daktarin $^{\circledR}$, em ratos, e verificou-se que o gel de C. Citratus teve eficacia correlata ao gel Daktarin ${ }^{\circledR}$.

Ao observar compostos específicos do óleo do C. citratus, como o citral (principal componente - 80\%), também há resultados significativos, numa correlação composto versus medicamento, como evidenciam Sousa et al. (2016). Os autores relatam que, o citral pode ter efeito sinérgico ao fluconazol em isolados de Candida, contribuindo na terapia, e proporcionando novas possibilidades de tratamento contra Candida. Além disso, demonstrou que provavelmente o efeito antifúngico provem deste composto, porém não obteve resultado sobre o modo de ação deste efeito, refutando sua hipótese de que o citral morfológico à membrana das células provocaria efeito ao ligar-se a membrana do ergosterol.

Ratificando esta evidência, Chatrath et al. (2019), demonstraram que o citral tem efeito significativo contra Candida, principalmente sobre cepas de Candida tropicalis $(32 \mathrm{ug} / \mathrm{ml})$, como também, tem efeito inibitório na formação do biofilme in vitro (numa concentração de $64 \mathrm{ug} / \mathrm{ml}$ ), em superfícies abióticas, causando danos interpostas no biofilme. No entanto, demonstrou-se que o citral aumentou a produção de espécies reativas de oxigênio in vitro. Observa-se também sua relação com o citocromo P-450 e com o ERG11 (lanosterol 14-dimetilase), no qual, há um aumento na expressão dessas enzimas, indicando a indução do mecanismo de tolerância a drogas semelhante ao do fluconazol. Observado este efeito do citral, é importante evidenciar que é preciso cautela no uso do óleo essencial de C. citratus. Nessa perspectiva, Souza et al. (2019), 
salientam que o citral exibiu citotoxidade em concentrações de $5 \mathrm{mg} / \mathrm{ml}$ ou em concentrações superiores, o que provoca diminuição na viabilidade celular, em células HepG2, como também genotoxidade, levando a danos significativos ao DNA, mesmo em baixas concentrações e, devido ao potencial efeito mutagênico, pode, por consequência, levar ao surgimento do câncer bucal.

Ao avaliar a relação sobre o geraniol, em isolados da cavidade bucal, Leite et al. (2015), demonstram que esse composto apresentou CIM de $16 \mu \mathrm{g} / \mathrm{ml}$ quando interposto a $90 \%$ das cepas de C. albicans testadas, porém, não demonstrou de forma clara o mecanismo de ação, refutando uma possível ligação do geraniol ao ergosterol da membrana. No entanto, a partir dessa não relação com o ergosterol, observou-se capacidade de inibição de pseudo-hifas e formação de clamidoconídios. Observa-se também, segundo Queiroz et al. (2017) que o geraniol também apresenta citotoxidade, causando diminuição da viabilidade celular em células HepG2.

Quanto ao $\beta$-mirceno houve resultados evidenciando que o $\beta$-mirceno tem efeito citotóxico em variadas concentrações, em células HepG2 e leucócitos, como também, têm efeitos genotoxicos com danos significativos ao DNA e anormalidades cromossômicas significativas, podendo assim, estar associado a fatores carcinogênicos, e teratogênicos na mucosa bucal (Orlando, et al., 2019).

\section{Conclusão}

Os estudos mostram a eficácia da utilização do Cymbopogon citratus (DC.) Stapf no tratamento do gênero Candida, que afeta a cavidade bucal, principalmente como forma de complementação do tratamento. Entretanto, é necessário cautela para o uso, visto que pesquisas recentes apontam resultados indicativos de toxicidade com relação aos componentes que integram essa espécie vegetal.

Ressalta-se que a maioria dos estudos com C. citratus foram ensaios pré-clínicos in vitro, sendo fundamental a realização de mais estudos, incluindo pesquisas in vivo, com o propósito de viabilizar a produção de um futuro medicamento como alternativa de tratamento para combater a infecção fúngica causada pelo fungo Candida, principalmente na cavidade bucal.

Espera-se que o presente trabalho possa incentivar a realização de novos estudos, in vivo, do tipo caso-controle, observando a dose-resposta na utilização do óleo ou extrato essencial de $C$. citratus como tratamento contra a cepa de Candida na cavidade bucal, utilizando para isso, uma metodologia criteriosa e amostra significativa que possibilitará observar a efetividade do tratamento.

\section{Referências}

Almeida, R. B. A., et al (2008). Atividade antimicrobiana de Cymbopogon citratus (DC.) stapf sobre Candida spp. Revista. Odontologia UNESP, 37(2), 147153. https://www.revodontolunesp.com.br/article/588018447f8c9d0a098b4b48

Almeida, R. B. A., et al (2013). Antimicrobial activity of the essential oil of Cymbopogon citratus (DC) Stapf. on Staphylococcus spp., Streptococcus mutans and Candida spp. Revista Brasileira de Plantas Medicinais, (Botucatu), 15(4), 474-482. 10.1590/S1516-05722013000400002

Bhatti, M. A., et al (2018). Evalution of antibacterial and antifungal activities of cymbopogon citratus \& psidium guajava from Sialkot orogin. PharmacologyOnLine, 1, 155-163. https://www.researchgate.net/publication/325263205_Evaluation_of_antibacterial_and_antifungal_act ivities_of_cymbopogon_citratus_and_psidium_Guajava_from_Sialkot_origin

Boukhatem, M. N., Ferhat, M. A., Kameli, A., Saidi, F., \& Kebir, H. T. (2014). Lemon grass (cymbopogon citratus) essential oil as a potent anti-inflammatory and antifungal drugs. Libyan Journal of Medicine, 9, 25431.10.3402/LJM.V9.25431

Chatrath, A., Gangwar, R., Kumari, P., \& Prasad, R. (2019). In Vitro Anti-Biofilm Activities of Citral and Thymol Against Candida Tropicalis. Journal of Fungi, 5(1), 13. 10.3390/jof5010013

Crocco, E. I., et al (2004). Identificação de espécies de Candida e susceptibilidade antifúngica in vitro: estudo de 100 pacientes com candidíases superficiais. Anais Brasileiros de Dermatologia, 79(6), 689-97. 10.1590/S0365-05962004000600005 
Dutta, B. K., Karmakar, S., Naglot, A., Aich, J. C., \& Begam, M. (2007). Anticandidial activity of some essential oils of a mega biodiversity hotspot in India. Mycoses, 50(2), 121-124. 10.1111/j.1439-0507.2006.01332.x

Ekpenyong, C. E., \& Akpan, E. E. (2015). Use of Cymbopogon citratus essential oil in food preservation: Recent advances and future perspectives. Critical Reviews in Food Science and Nutrition, 57(12), 2541-2559. 10.1080/10408398.2015.1016140

Irkin, R., \& Korukluoglu, M. (2009). Effectiveness of Cymbopogon citratus L. essential oil to inhibit the growth of some filamentous fungi and yeasts. Journal of Medicine Food, 12(1), 193-197. 10.1089/jmf.2008.0108

Juniatik, M., Hidayati, K., Wulandari, F.P., Pangestuti, N., Munawaroh, N., Martien, R., \& Utami, S. (2017). Formulation of nanoemulsion mouthwash combination of lemongrass oil (cymbopogon citratus) and kaffir lime oil (Citrus hystrix) AGAINST Candida albicans ATCC 10231. Traditional Medicine Journal, 22(1), 7-15. 10.22146/tradmedj.24255

Khan, M. S., \& Ahmad, I. (2012). Biofilm inhibition by Cymbopogon citratus and Syzygium aromaticum essential oils in the strains of Candida albicans. Journal of Ethnopharmacology, 140(2), 416-423. 10.1016/j.jep.2012.01.045

Khosravi, A.R., Sharifzadeh, A., Nikaein, D., Almaie, Z., \& Gandomi, N.H. (2018). Chemical composition, antioxidant activity and antifungal effects of five Iranian essential oils against Candida strains isolated from urine samples. Journal of Medical Mycology, 28(2), 355-360. 10.1016/j.mycmed.2018.01.005

Koseki, Y., Tanaka, R., \& Murata, H. (2018). Development of antibacterial denture cleaner for brushing containing tea tree and lemongrass essential oils. Dental Materials Journal, 37(4), 659-666. 10.4012/dmj.2017-295

Leite, M. C., De Brito Bezerra, A. P, De Sousa, J. P., \& De Oliveira L.E. (2015). Investigating the antifungal activity and mechanism(s) of geraniol against Candida albicans strains. Medical Mycology, 53(3), 275-284. 10.1093/mmy/myu078

Lemos, G. C. S., Santos, A. D., Freitas S. P., \& Gravina, G. A. (2013). Controle de plantas invasoras em cultivo orgânico e convencional de capim-limão (Cymbopogoncitratus). Revista Brasileira de Plantas Medicinais, 15(3), 405-414. 10.1590/S1516-05722013000300014

Machado, A.C., \& Oliveira, R. (2014). Medicamentos Fitoterápicos na odontologia : evidências e perspectivas sobre o uso da aroeira-do-sertão (Myracrodruon urundeuva Allemão ). Revista Brasileira de Plantas Medicinais, 16(2), 283-289. 10.1590/S1516-05722014000200018

Madeira, P. L. B., et al (2016). In vitro Effects of Lemongrass Extract on Candida albicans Biofilms, Human Cells Viability, and Denture Surface. Frontiers in Cellular and Infection Microbiology, 6, 71.10.3389/fcimb.2016.00071

Melo, I. A., \& Guerra, R. C. (2014). Candidíase oral: um enfoque sobre a estomatite por prótese. Salusvita, 33(3), 389-414. https://www.researchg ate.net/publication/270498018_candidiase_oral_um_enfoque_sobre_a_estomatite_por_protese

Mendes, K. D. S., et al (2008). Revisão integrativa: método de pesquisa para a incorporação de evidências na saúde e na enfermagem. Texto-contexto Enfermagem, 17(4), 758-764. 10.1590/S0104-07072008000400018

Miranda-Cadena, K., Marcos-Arias, C., Mateo, E., Aguirre, J. M., Quindós, G., \& Eraso, E. ( 2018). Prevalence and antifungal susceptibility profiles of Candida glabrata, Candida parapsilosis and their close-related species in oral candidiasis. Archives of Oral Biology, 95, 100-107. 10.1016/j.archoralbio.2018.07.017

Orlando, J. B., et al (2019). Genotoxic effects induced by beta-myrcene following metabolism by liver HepG2/C3A human cells. Journal of Toxicoogyl and Environmental Health, Part A, 82(3), 176-185. 10.1080/15287394.2019.1577195

Pavithra, J. A., Srinikethan, G., Shubhada, C., Pradeep, K., Gopala, M., Kulkarni, R., \& Praveenchandra, Kr. (2014) .Effect of five plant extracts on adhesion of Candida albicans onto human buccal epithelial cells: an in-vitro study. Egyptian Pharmaceutical Journal, 13(2), 137. 10.4103/1687-4315.147089

Pereira, A. S., et al (2018). Metodologia da pesquisa científica. UFSM. https://repositorio.ufsm.br/bitstream/handle/1/15824/Lic_Computacao_MetodologiaPesquisa-Cientifica.pdf?sequence $=1$

Pokpong, A., Suwan, C., \& Theerathavaj, S. (2014). Lemongrass-Incorporated Tissue Conditioner Against Candida albicans Culture. Journal of clinical and diagnostic research : JCDR, 8(7), ZC50-ZC52. 10.7860/JCDR/2014/8378.4607

Prakash, S., Ramasubburayan, R., Ramkumar, V. S., Kannapiran, E., Palavesam, A., \& Immanuel, G. (2016). In vitro-scientific evaluation on antimicrobial, antioxidant, cytotoxic properties and phytochemical constituents of traditional coastal medicinal plants. Biom Pharmaceuticals, 83, 648-657. 10.1016/J.BIOPHA.2016.07.019

Queiroz, T. B., et al (2017). Cytotoxic and genotoxic potential of geraniol in peripheral blood mononuclear cells and human hepatoma cell line (HepG2). Genetics and Molecular Research, 16(3). 10.4238/gmr16039777

Sharifzadeh, A., Khosravi, A. R., Shokri, H., Asadi Jamnani, F., Hajiabdolbaghi, M., \& Ashrafi Tamami, I. (2013). Oral microflora and their relation to risk factors in HIV+patients with oropharyngeal candidiasis. Journal of Medical Mycology, 23(2), 105-112. 10.1016/j.mycmed.2013.02.001

Silva, C. B., Guterres, S. S., Weisheimer, V., \& Schapoval, E. R. S. (2008). Antifungal activity of the lemongrass oil and citral against Candida spp. Brazilian Journal of Infectious Diseases, 12(1), 63-66. 10.1590/S1413-86702008000100014

Silva, N. B., et al (2017). Antifungal Activity of the Essential Oil of Cymbopogon citratus (DC) Stapf. An in vitro study. Journal of Oral Research, [S.1.], 6(12), 319-323. 10.17126/joralres.2017.092

Sousa, J. P., et al (2016). Antifungal Activity of Citral by Disruption of Ergosterol Biosynthesis in Fluconazole Resistant Candida tropicalis. International Journal of Tropical Disease \& Health, 11(4), 1-11. 10.9734/IJTDH/2016/21423

Souza, A. C. S., et al (2019). Citral presents cytotoxic and genotoxic effects in human cultured cells. Drug and Chemical Toxicology, 43 (4), 2019. 
Research, Society and Development, v. 10, n. 3, e13010312844, 2021

(CC BY 4.0) | ISSN 2525-3409 | DOI: http://dx.doi.org/10.33448/rsd-v10i3.12844

\section{$10.1080 / 01480545.2019 .1585445$}

Spalanzani, R. N., et al (2018). Clinical and laboratorial features of oral candidiasis in HIV-positive patients. Revista da Sociedade Brasileira de Medicina Tropical, 51(3), 352-356. 10.1590/0037-8682-0241-2017

Swetha, T., Peter, D., Mccall, A., Hwang-Wong, E., Rojas, I.G., Cormack, B., \& Edgerton, M. (2016). Candida glabrata Binding to Candida albicans Hyphae Enables Its Development in Oropharyngeal Candidiasis. PLoS Pathogens, 12(3), 1-21. 10.1371/journal.ppat.1005522

Taweechaisupapong, S., Aieamsaard, J., Chitropas, P., \& Khunkitti, C.W. (2012). Inhibitory effect of lemongrass oil and its major constituents on Candida biofilm and germ tube formation. South African Journal of Botany, 81, 95-102. 10.1016/j.sajb.2012.06.003

Taweechaisupapong, S., Ngaonee, P., Patsuk, P., Pitiphat, W., \& Khunkitti, W. (2012). Antibiofilm activity and post antifungal effect of lemongrass oil on clinical Candida dubliniensis isolate. South African Journal of Botany, 78, 37-43. 10.1016/j.sajb.2011.04.003

Taweechaisupapong, S., Boonmars. T., Satthanakul, P., Chatchanayuenyong, V., Nitinon, S., Soodtoetong, U., Thipsongkroh, W., \& Khunkitti1, W. (2018). Effect of lemongrass gel against Candida albicans in rat model of oral candidiasis. Songklanakarin Journal of Science and Technology, 42(2), 299-304. $10.1456 /$ sjst-psu.2020.39

Tyagi, A. K., \& Malik, A. (2010). Liquid And Vapour-Phase Antifungal Activities Of Selected Essential Oils Against Candida Albicans: Microscopic Observations And Chemical Characterization Of Cymbopogon Citratus. Bmc Complementary And Alternative Medicine, 10, 65. 10.1186/1472-6882-10-65 